\title{
Congestion Control in Wireless Sensor Networks Using Genetic Algorithm
}

\author{
Navid Samadi Khangah \\ Department of Computer \\ Engineering, Tabriz Branch, Islamic \\ Azad University Tabriz, Iran
}

\author{
Ali Ghaffari \\ Department of Computer \\ Engineering, Tabriz Branch, Islamic \\ Azad University Tabriz, Iran
}

\begin{abstract}
Sensor network consists of a large number of small nods, strongly interacting with the physical environment, takes environmental data through sensors, and reacts after processing on information. Wireless network technologies are widely used in most applications. As wireless sensor networks have many activities in the field of information transmission, network congestion cannot be thus avoided. So it seems necessary that some new methods can control congestion and use existing resources for providing better traffic demands. Congestion increases packet loss and retransmission of removed packets and also wastes of energy. In this paper, a novel method is presented for congestion control in wireless sensor networks using genetic algorithm. The results of simulation show that the proposed method, in comparison with the algorithm LEACH, can significantly improve congestion control at high speeds.
\end{abstract}

Keywords: wireless sensor networks, congestion control, genetic algorithm, optimization, clustering.

\section{INTRODUCTION}

Wireless sensor networks are networks including independent sensors in environment which measure physical or environmental conditions such as temperature, sound, vibration, pressure, motion or pollutants in different locations. These sensors are small and work interacting with another and have a limited amount of stored energy, amount of memory and bandwidth. Restrictions such as buffer memory, limited computing, stored electric power have caused to be proposed several methods for routing and data transmission. Congestion in wireless sensor networks occurs when sensor nodes receive more number of packets than the number they can send. Therefore, it is necessary to use fast and efficient congestion control mechanisms in wireless sensor networks [1]. Therefore, transport layer in wireless sensor networks is responsible for controlling congestion. Congestion control methods are done by the two methods: traffic and resource control. Congestion not only causes loosing severe information, but also leads to excessive consumption of energy in the nodes. Many multicast routing protocols are provides for data transmission in multiple paths, however congestion control mechanisms are rarely to be found for multiple routing. In this paper, a congestion control protocol is provided for wireless sensor networks using genetic algorithms aimed at increasing reliability and lifetime level of the network and the high throughput.

\section{CONGESTION CONTROL}

There are generally two reasons for Congestion in wireless sensor networks. The first reason is that packet arrival rate is much more of packet service rate. This mode happens more to nodes near the destination node, because they usually carry more upward combined traffic. The second reason is due to aspects of performance in level link such as competition, interference and bit error rate. Congestion in wireless sensor networks has a direct impact on energy efficiency and quality of service. For example, congestion can lead to a buffer overflow, hence to a larger queue delay and losing more packets. Packet loss not only leads to reduce reliability and quality of service but also squandering the nodes limited energy. Thus, congestion in sensor networks should be controlled efficiency by avoiding congestion occurring, or reducing the congestion. Several congestion control techniques are proposed for wireless sensor networks [2][3][4]. All the congestion control mechanisms have the same basic goal: All of them first try to diagnose or detect congestion, and after detection of congestion are too aware other groups of the situation of congestion. In general, there are three phases for congestion control: Congestion detection phase, Congestion notification phase and rate adjustment phase [5][6].

\section{RELATED WORKS}

Generally different protocols are introduced for transport layer in wireless sensor networks so that each is able to effectively control Congestion. Among the proposed protocols, there are some protocols that control just congestion controlling and some guarantee just reliability. There are rare number of these protocols which can both ensure and control the reliability and congestion.

SRCP (Sensor Reliability and Congestion Control Protocol) algorithm determines traffic, based on increasing the packet send process and decreases throughput in a short time. This is a rate based protocol which adjusts distance of sending packets after a fixed time, called track time [7]. Mesh interface algorithm, as network topology input, uses desired sent rate flow for routing directions. Imaging the network interference, as a conflict diagram, is approximate and dependent using an iterative process in order to estimate (fair max-min) secure transmission rate for each flow to reflect the total network throughput [8]. SPEED algorithm tries to maintain the desired speed in real time and does the tasks uniformly by providing applications. Traffic diversion causes end-to-end delay to be proportional to the distance between the source and destination through multiple paths and adjust the transmission rate [9]. HTAP (Hierarchical Tree Alternative Path) algorithm tries to guarantee reliability of applications during the period of overloading (overload) without reducing the funds rate at the time of important events. HTAP which is a combination of two algorithms: creation of alternative route, creation of a hierarchical tree, chooses it by using the network congestion [10]. TARA (Thermal-Aware Routing Algorithm) algorithm defines total link congestion to be as the total of traffic and traffic interference links and selects bottleneck the large amounts of congestion as well [11]. WCCP (WMSN Congestion Control Protocol) algorithm is a two-part protocol. In its resources sector, SCAP is used to set transmission rate and distribution of abandoned pockets. The aim of SCAP 
protocol, at the first, is to avoid the congestion from the source node. In receiver, RCCP protocol is used for detecting the congestion occurrence and informing the source nodes in the regarding congestion [12]. LEACH (Low Energy Adaptive Clustering Hierarchy protocol) is self-organizing clustering protocol which distributes energy load on the network sensors. In LEACH, nodes organize themselves into local clusters so that a node acts in the cluster as the cluster head [13].

\section{THE PROPOSED ALGORITHM}

Before a genetic algorithm can be executed first must be found a suitable encoding (or representation) for intended problem intended. The usual manner of representing chromosomes in genetic algorithm is a binary string. Each decision variable becomes a binary form and then is created chromosome by getting together the variables. Although this method is a method of encoding extended but other ways are growing such as representation by real numbers. A fitness function must be also devised to attribute a value to each coded solution. During the run, parents are selected for breeding and, using crossover or mutation operators, are combined together to produce new children. This process is repeated several times to produce the next generation population. Then this population is investigated and if the convergence criteria are met, the above process is terminated. Work process in genetic algorithm is done by two mutation and integration methods. Work process of integration method is studied in the proposed algorithm as follows.

First the nodes coordinate are named and the number of nodes is identified. The number of nodes is defined as nnodes. Then the nodes are put in a variable named $k$. The variable $k$ is divided into two parts $k_{1}$ and $k_{2}$. By selecting the number of nodes, an amount of primary energy is given to each of them (initial amount of energy is between 0 and 1). After this step in every time of overall execution, genetic algorithm is executed as much as 10 times (the number 10 is the numerical value of the option).

\subsection{Stages of integration implementation}

Coordinate nodes are considered 8-bit, $k_{1}$ and $k_{2}$ are the integer numbers converted to binary and placed in new variables $k_{3}$ and $k_{4} \cdot k_{3}$ and $k_{4}$ are the same chromosomes in the proposed algorithm. At this stage the variables $C, D$ are defined in order to locate chromosomes which ultimately are obtained from creation of chromosomes positions (coordinate) of nodes. To perform the integration, the cut takes place randomly into chromosomes in each time intercourse so that the state spatial coordinates (chromosomes) are improved by this process. have a 9-point text, as you see here. Please use sans-serif or non-proportional fonts only for special purposes, such as distinguishing source code text. If Times Roman is not available, try the font named Computer Modern Roman. On a Macintosh, use the font named Times. Right margins should be justified, not ragged.

\subsection{Stages of implementation mutation}

There is a variable called $m$ in mutation randomly chosen to practice jumps and again the chromosomes are placed in variables $A$ and $B$. By examining $m$, if $A m=0$, the values one are put at chromosome home, otherwise zero. Mutation output will be as integration in $C$ and $D$. After combination and mutation, cluster operations are carried out. For selecting cluster head, the amount battery is used, while for proper functioning, the position of the nodes, i.e., the node distance. The formular for selecting cluster head is as follows:

$$
\text { Temp }=\frac{p}{1-p^{*}\left(r \bmod \frac{1}{p}\right)}
$$

Where $p$ is the probability cluster head node is and placed equal 0.1 and $r$ the current stage.

In case of holding the cluster head, for calculating the distance of the nodes coordinates from the sink, equation (2) is used [14]:

distance $=\sqrt{\left(E_{i}-E_{\text {nnodes }+1}\right)^{2}+\left(F_{i}-F_{\text {nnodes }+1}\right)^{2}}$

where $E_{i}, E_{\text {nnodes }}, F_{i}, F_{\text {nnodes }}$ denote, respectively, coordinates of nodes, number of nodes in $X$ axis, the coordinates of nodes, and the number of nodes in $y$ axis. Before the genetic operations, a random amount of primary energy is assigned to each node. Here (in GA) the distance of the node to sink is initially examined for required processing (operation) in order to assign the amount of energy which a node needs in a specified distance between the node and sink. After determining the amount of primary energy for each node in the new population, the aim of the proper functioning of the proposed algorithm is described in the sequel. Nodes distance ratio to sink and primary energy nodes (obf) is obtained by equation (3):

$\mathrm{obf}=\sqrt[2]{\frac{\left(E_{i}-E_{\text {nnodes }+1}\right)^{2}+\left(F_{i}-F_{\text {nnodes }+1}\right)^{2}}{\operatorname{Intionl} E(1, i)}}$

where $E_{i}, E_{n n o d e s}, F_{i}, F_{n n o d e s}$, are as before, IntionlE $(1, i)$ is initializing in matrix $1 \times i$ and $i$ is the number of nodes. All of the values obtained by the above ratios are placed in a variable called sum. To the number of nodes, a new variable, named prob, in order to accommodate the matrix of relationships which can save matrix values is established in prob variable. Appropriate matrix values are good in sum of the values. The first entry of the prob matrix is stored in prob variable, as a minimum, to be designed by finding prob matrix global minimum so that in the end, according to the above studies, a new state space (coordinates) is established for sink.

\section{THE IMPLEMENTATION RESULTS}

In this section the results obtained by the implementation of the proposed method are evaluated. MATLAB software is used for evaluating the proposed method whereas LEACH algorithm for comparing. Simulation parameters are specified in Table 1.

Table 1. Table captions should be placed above the table

\begin{tabular}{|l|l|}
\hline Parameters & Value \\
\hline Number of nodes & $50,100,150,200$ \\
\hline Environment size & $100 \mathrm{~m}^{*} 100 \mathrm{~m}$ \\
\hline Sink localization & $(50 * 50)$ \\
\hline Energy model & $0.5 \mathrm{~J}$ \\
\hline Rounds & $500,1000,1500,2000$ \\
\hline
\end{tabular}

The state space of nodes is displayed as in Figures 2-5 in LEACH and LECH_GA algorithms, respectively. 


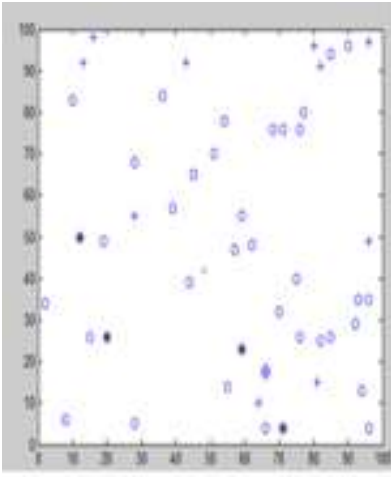

IEACH_GA

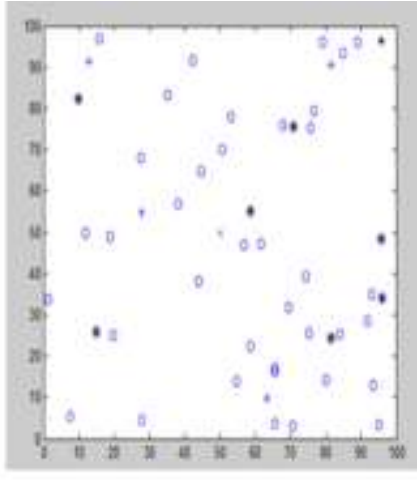

LEACH
Figure. 2 The state space for LEACH and LECH_GA algorithms with the number of 50 nodes and 500 rounds

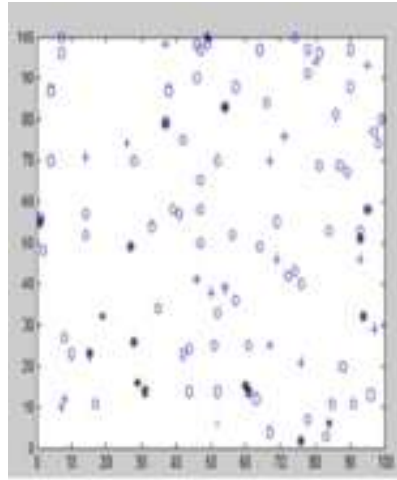

IEACH GA

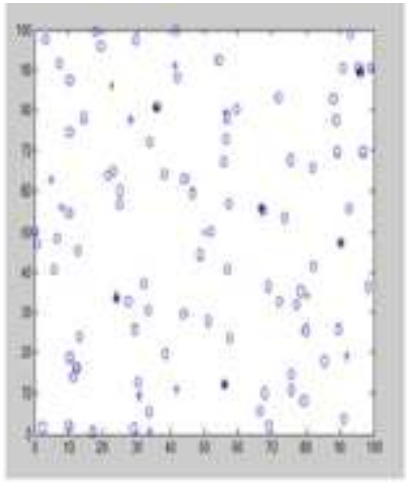

LEACH
Figure. 3 The state space for LEACH and LECH_GA algorithms with the number of 100 nodes and 1000 rounds

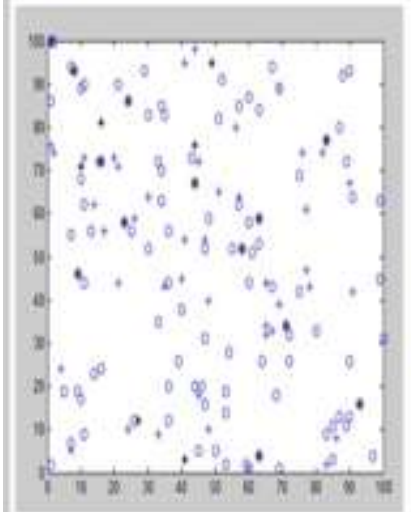

LEACH GA

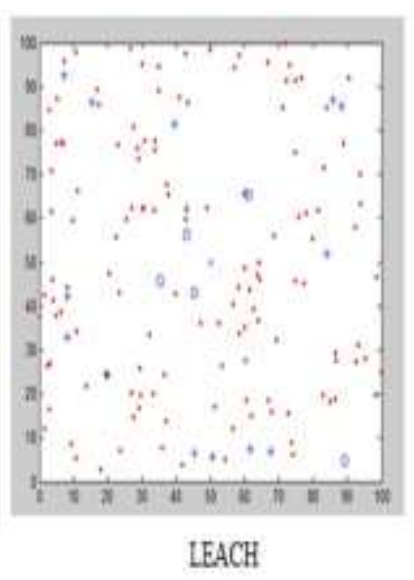

LEACH
Figure. 4 The state space for LEACH and LECH_GA algorithms with the number of 150 nodes and 1500 rounds

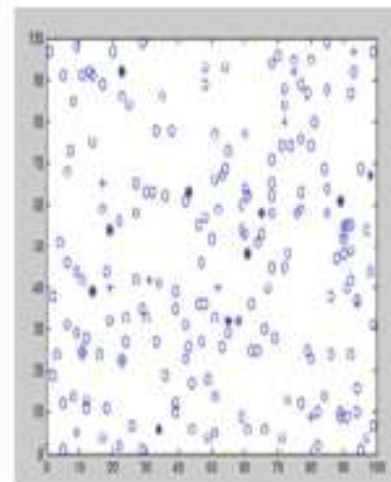

LEACH GA

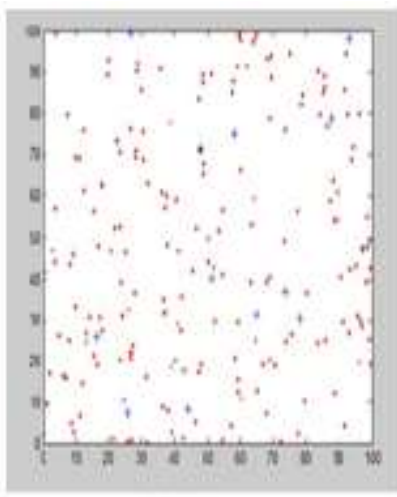

LEACH
Figure. 5 The state space for LEACH and LECH_GA algorithms with the number of 200 nodes and 2000 rounds

Figure 6 compares the dead nodes for (LEACH GA) and (LEACH) algorithms. In around 500, the number of dead nodes in both algorithms is zero. In around 1000 onwards, the dead nodes in LEACH algorithm dramatically increased, but this amount is zero for LECH_GA algorithm. Due to the high number of rotations, the implementation time of the algorithm becomes longer and dead nodes largely increase in LEACH algorithm, and genetic algorithms due to having more energy, still do not have any dead nodes.

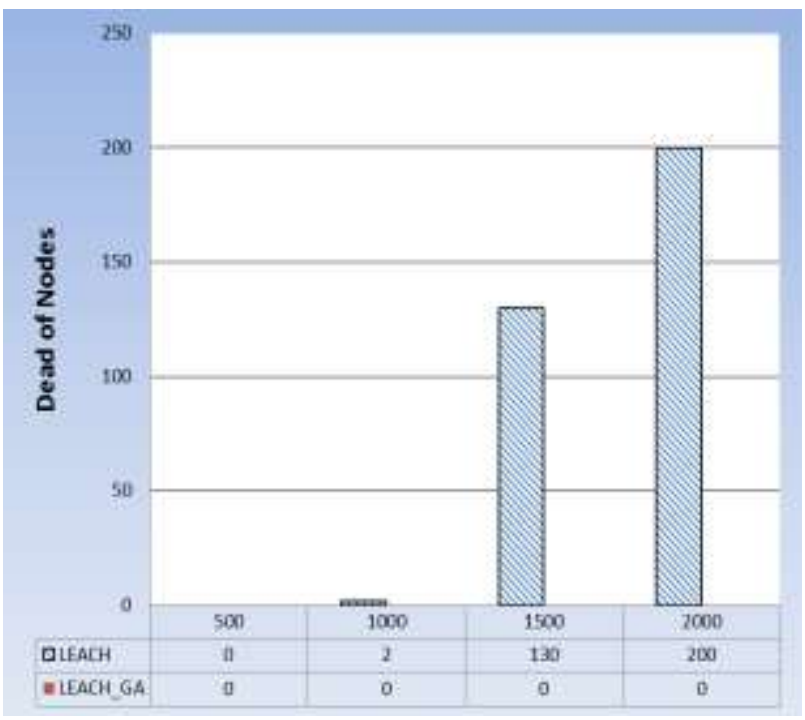

Figure. 6 Comparison of dead nodes in the proposed algorithm and LEACH algorithm

Figure 7 shows the comparison of ripe nodes to destination (base station) in the two genetic algorithms LECH_GA and $\mathrm{LEACH}$. According to Figure 7, in period 500 the number of incoming packets in LEACH and LECH_GA algorithms are respectively 2508 and 7412 . By increasing the number of rounds, the number of packets received to the destination will also increase. This is due to changes in sink state space, and the position of the nodes by the proposed method and there is a significant difference in the number of rotations. On the other hand in LEACH algorithm by increasing the number of turns, live nodes decrease and markedly decrease the packets ripen to the destination. 


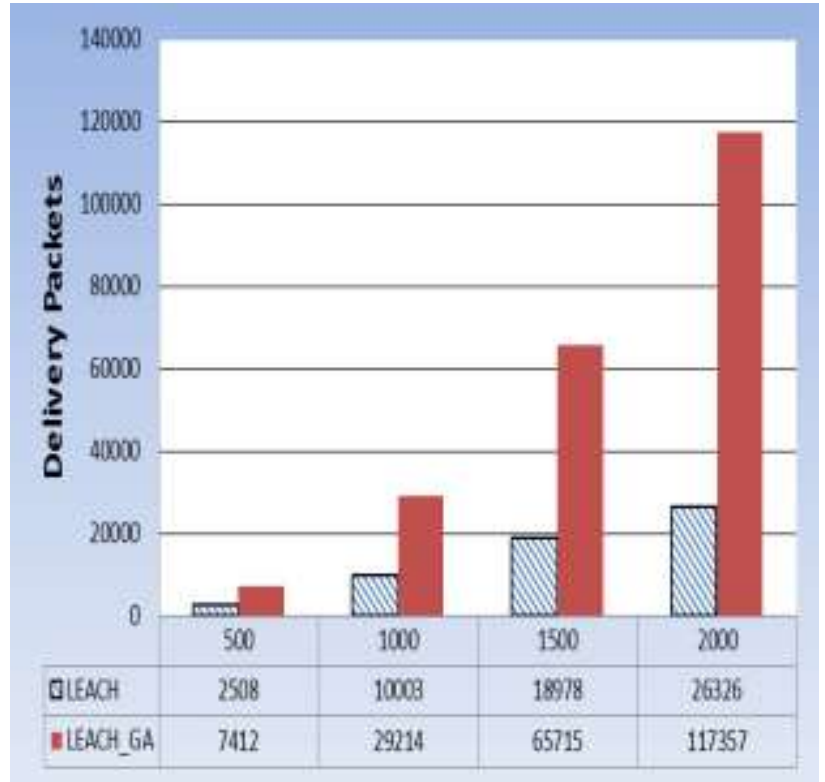

Figure. 7 The number of incoming packets to sink

Figure 8 shows the comparison of the number of choosing cluster in the two genetic algorithms LECH_GA and LEACH. In lower revs in the genetic algorithm, no need to select much cluster, according to the production chromosomes. Also it is observed that by increasing the rounds, due to high energy consumption, cluster head with high reliability is chosen for packet transmission in genetic algorithms.

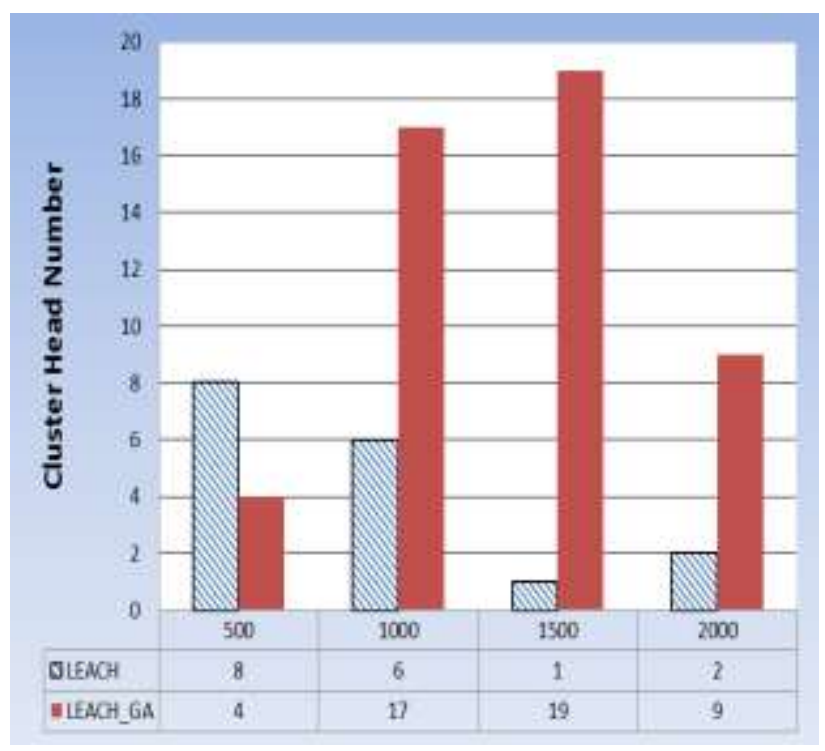

Figure. 8 Selection of cluster head

Figure 9 shows the comparison of energy consumption for genetic algorithm, LEACH-GA and LEACH. Energy consumption for the proposed algorithm has not so declined, but energy consumption for LEACH algorithm has ended in a round 2000 .

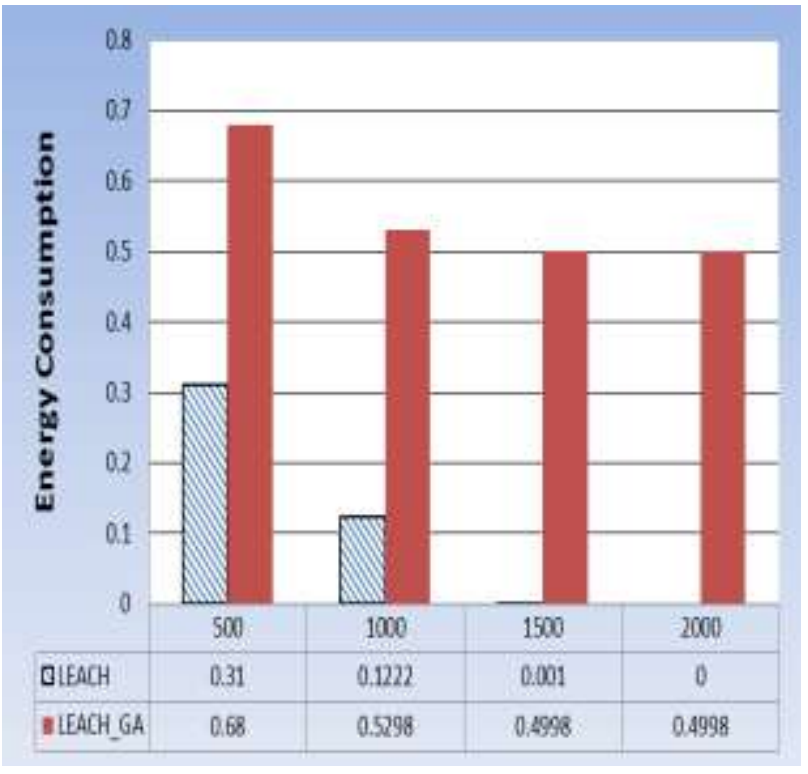

Figure. 9 The remaining energy consumption in both LEACH and LEACH_GA algorithms

\section{CONCLUSION}

In this article, congestion control was studied using genetic algorithms in wireless sensor networks. According to the results, it was given a new value for the state space of the sink so that it can have good performance at sending incoming packets to the destination (sink) when the position (state-space) of the sink changes, reducing nodes losses, traducing energy consumption power, selecting better cluster. Whatever the number of the terms is more in the proposed algorithm, the performance in terms of dead nodes, number of incoming packets to the destination and energy would be highly better. A deep understanding of congestion control lets the transmission comprehensive protocol design with reliability. Addressing the transport layer with high reliability is essential to ensure effectiveness of the applications. Checking the transfer protocols shows controls of the reliability of a vision to this task (congestion control).

\section{REFERENCES}

[1] Akyildiz, I. F., Su, W., Sankarasubramaniam, Y. 2002. Computer Networks: The International Journal of Computer and Telecommunications Networking archive Volume 38 Issue 4, 393-422.

[2] Tao, Q. and Yu, F. Q. 210. ECODA: Enhanced Congestion Detection and Avoidance for Multiple Class of Traffic in Sensor Networks. in proc. IEEE conf.

[3] Vijayaraja, V. and Hemamalini, R. Congestion in Wireless Sensor Networks and Various Techniques for Mitigating Congestion - A Review. in proc. IEEE conf. May2010.

[4] Sridevi, S. Usha, M. and Pauline, G. 2012. Priority Based CongestionControl For Heterogeneous Traffic In Multipath Wireless Sensor Networks. inproc. IEEE conf.

[5] Hull, b. jamieson, k. and Balakrishnan, h. 2004. Mitigation congestion in wireless sensor networks. in procedding of ACM sensys, vol.4, USA,

[6] Wang, C. and Sohraby, K. 2006. A Survey of Transport Protocols for Wireless Sensor Networks. IEEE Network, vol.20, pp.34-40, 
[7] Tezcan, N., and Wang, W. 2007. ART: an asymmetric and reliable transport mechanism for wireless sensor networks. Int. J. Sen. Netw, 2(3), 188-200.

[8] Sundaresan, K., Anantharaman, V., Hsieh, H. Y., Sivakumar, R. 2005. ATP: A Reliable Transport Protocol for Ad Hoc Networks. IEEE Trans. Mobile Computing, 4(6), 588603.

[9] Kang, J., Zhang, Y., Nath, B. 2007. TARA: TopologyAware Resource Adaptation to Alleviate Congestion in Sensor Networks. IEEE Trans. Parallel Distrib. Syst, 18(7), 919-931. [10] Wang, G., \& Liu, K. 2009. Upstream hop-by-hop congestion control in wireless sensor networks. in Proc. IEEE 20th Int. Symp. on Personal, Indoor and Mobile Radio Communications, 1406-1410.

[11] Monowar, M., Rahman, M., Hong, V. S. 2008. Multipath Congestion Control for Heterogeneous Traffic in Wireless Sensor Network, in Proc. 10th Int. Conf. on Advanced Communication Technology, ICACT, 3, 1711-1715.

[12] Mahdizadeh Aghdam, S., Khansari, M. , Rabiee, H. R., Salehi, M. 2014. WCCP: A congestion control protocol for wireless multimedia communication in sensor networks. Ad Hoc Networks 13 , 516-534.

[13] Gou, H., Yoo, Y., Zeng, H. 2009. A Partition-Based LEACH Algorithm for Wireless Sensor Networks. CIT '09. Ninth IEEE International Conference on, Computer and Information Technology, 2009, IEEE, PP. 40-45.

[14] Feigenbaum, J., Kannan, S., McGregor, A., Suri, S. and Zhang, J. 2005. Graph distances in the streaming model: the value of space. In ACM-SIAM Symposium on Discrete Algorithms, 745-754. 